\title{
ORACIONES DE RELATIVO COMO MODIFICADORES REFERENCIALES DE UN GRUPO NOMINAL
}

Resumen. En esta aportación se contempla la variación del artículo en los grupos nominales GN modificados por una oración de relativo. Se intenta descubrir rasgos gramaticales de la oración relativa que decidan acerca de la referencia del conjunto nominal. Este estudio de tipo estructural pretende llegar a profundizar en los mecanismos que rigen el funcionamiento del artículo, principal exponente de la (in)definitud en castellano.

Palabras clave: definitud, grupo nominal, oración de relativo.

\section{Variación del artículo en el antecedente de una oración de relativo: preliminares}

Las reglas de uso del artículo español son complicadísimas, en comparación no solo con las lenguas germánicas, sino también las románicas. En sus mecanismos de empleo juegan un papel importante múltiples factores de diversa índole (Iturrioz, 1996: 339-352). En esta aportación pretendemos dar cuenta de la variación del artículo en los grupos nominales complementados por oraciones de relativo con antecedente expreso, problema que fue debatido por lingüistas como Hawkins (1978), Kleiber (1981) o Lyons (1999). Hay que poner de relieve una mayor frecuencia de las oraciones de relativo en los textos españoles que en los de otras lenguas. Dada la desaparición del participio activo latino (cantante), no es recomendable construir grupos nominales con este modificador. Compárense, p. ej., The girls sitting over there are trying to impress you Las muchachas que están sentadas allí tratan de impresionarte.

Como es sabido, «Las [oraciones] relativas que tienen antecedente expreso ejercen una función similar a la de los demás modificadores del

* Uniwersytet im. Adama Mickiewicza w Poznaniu. 
sustantivo. Así, la oración subrayada en una historia que muy bien puede no haber ocurrido desempeña la misma función que los modificadores adjetivales subrayados en una historia imaginaria o una historia de ficción» (MNGLE, 2010: 838). Al igual que los adjetivos, las oraciones de relativo son capaces de restringir la extensión del GN que complementan (especificativas) o solo agregarle cierta información suplementaria no discriminativa (explicativas). Solo las oraciones especificativas pueden influir por sí mismas en la referencia del GN que complementan y, por lo tanto, son las construcciones que sometemos a examen en las líneas siguientes.

Considérese la distinta presentación del sintagma WOMAN WHO..., como determinado o indeterminado, en función de la información proporcionada por la subordinada adjetiva (vid. Hawkins, 1978: 133):

A woman who some sailor dated last night spread nasty rumours about Bill.

The woman who Bill dated last night spread nasty rumours about him.

Es un hecho que también una misma construcción puede manifestar dos variantes del determinante en función del contexto comunicativo existente entre los interlocutores. Así, por ejemplo:

Me enamoré de una desconocida que vi ayer en el cine.

Zakochałem się w takiej nieznajomej, którą widziałem wczoraj w kinie.

Lo indefinido del antecedente se puede representar en polaco mediante los determinantes taki, takiego rodzaju. En este caso, el hablante sabe bien que no está en condiciones de identificar en primera mención a la entidad desconocida por medio de una oración adjetiva porque la información que proporciona no evoca ninguna situación conocida en el oyente. Pero si el oyente del último ejemplo tuviera un conocimiento previo de desconocida que vi ayer en el cine, el hablante lo aprovecharía formulando un mensaje identificador con el artículo definido (AD), es decir:

Me enamoré de la desconocida que vi ayer en el cine.

Zakochałem się w tej nieznajomej, którą widziałem wczoraj w kinie.

El demostrativo tej, muy próximo en su significado al AD, subraya en polaco que el oyente está enterado acerca de un individuo concreto descrito de esta manera (Pawlik, 2001: 86-87). Dicho de otro modo, la diferencia referencial que se perfila mediante los determinantes polacos ten i taki suele expresarse en español a través de la lectura identificadora (ing. identifying) y calificativa (ing. classifying) de un conjunto nominal. En el segundo caso se actualiza el sustantivo, respondiendo a la pregunta CUÁL (desconocida) y en el primero, QUÉ o QUÉ TIPO DE (vid. NGLE, 2010: 467). 


\section{Oración relativa y la (in)definitud del antecedente}

Como advierten los autores del MNGLE (2010: 269),

Los USOS ENDOFÓRICOS del artículo determinado son aquellos en los que algún modificador o complemento interno del GN aporta la información necesaria para que este pueda emplearse como definido en su primera mención. Así pues, en [...] $Y$ entonces Olga le dio la carta que había estado escribiendo durante toda la mañana, la oración de relativo contiene la información necesaria para realizar la identificación (USO ENDOFÓRICO).

Este planteamiento no sintoniza con lo que declara $A$ Comprehensive Grammar of the English Language, según la cual este uso del artículo definido debe clasificarse como efecto de la catáfora (Quirk, 1985: 269).

No toda oración de relativo logra identificar su GN antecedente, lo cual infiere su indefinitud. La variación entre artículo definido (AD) e indefinido (AI) constituye uno de los mayores problemas que encara un hablante de ELE. Obsérvese cómo un mismo GN puede alternar distintos operadores referenciales con bastante libertad (vid. Morimoto, 2011: 59). Consideremos dos versiones españolas de una misma frase inglesa tomada del libro Time Machine que varían solo en el artículo:

El descenso lo realicé mediante (los) barrotes metálicos que salían de las paredes del pozo1.

En efecto, como se recordará, no siempre hay tanta soltura para conceder a un referente así modificado diferentes grados de determinación. Por ejemplo, si se oye decir una secuencia como:

Con los conflictos bélicos que hemos sufrido en nuestra historia republicana tenemos suficiente,

se debe percibir el sintagma los conflictos bélicos que hemos sufrido como reconocido y consabido, con rasgo de definitud obligatorio. En consecuencia, la omisión del determinante $\left({ }^{*}\right.$ Con conflictos bélicos que...) no encaja con claridad en un contexto pragmático. Sería de gran interés, por ende, señalar los rasgos estructurales y semántico-pragmáticos de las construcciones que admiten doble interpretación y las que exigen una única forma de determinación, como se aprecia en:

Me enamoré de unas / las desconocidas que vi ayer en el cine.

Me enamoré de los libros que ha escrito Borges hacia finales de su vida².

${ }^{1}$ No se percibe aquí un cambio de referencia importante.

${ }^{2}$ El cuantificador unos libros... no transmite el mismo comunicado, ya que se refiere solo a una parte de los libros comentados (contraste de cantidad). 
En resumen, nos encontramos con dos clases de construcciones que han de tomarse en cuenta: los casos de uso forzoso de un solo artículo correcto (AD o AI) o los de uso potestativo (a veces con distintas implicaciones) (vid. Leonetti, 1999: 89-90). Por razones de claridad metodológica nos proponemos un análisis enfocado tan solo en grupos nominales plurales (sustantivos contables, descuidados en estudios anteriores) y subordinadas de relativo introducidas únicamente por el pronombre relativo que. Siendo así, nuestra tarea consistiría en seleccionar debidamente entre el operador definido LOS / LAS y su correlato nulo, portador de indefinitud. Veremos en qué medida el contenido estructural de la subordinada impone a su antecedente la interpretación deseada. Nuestra elaboración tiene parcialmente un carácter experimental y se apoya en textos recogidos en el corpus CREA. Otros factores que desatendemos en nuestro estudio son el contexto oracional en que se encuentra un GN así complementado (sujeto, objeto, atributo, etc.) y el carácter genérico o no genérico del antecedente nominal.

\section{Algunos factores estructurales de la oración relativa y la interpretación del GN}

Nuestro propósito es descubrir rasgos estructurales, sobre todo sintácticos, de las oraciones relativas que favorezcan distintas interpretaciones del GN modificado. Por esta razón Hawkins (1978) y Kleiber (1981) plantean un reparto de las relativas, respectivamente en 'establishing non-establishing' y 'spécifiantes non spécifiantes'3 Hawkins (1978: 136) explica que «It seems that establishing relatives must relate the new, definite referent to some previously known object, or to participants in the talk-exchange, or to objects in the immediate situation». Kleiber (1981: 219), por su parte, asevera que «Les prédicats non spécifiants, qui sémantiquement décrivent une propriété, un attribut du référent, n'implique aucune localisation externe, aucun point de référence spatio-temporel». Tomándolo en cuenta podemos suponer que las subordinadas de relativo con estructura copulativa son las que tienden a reclamar un GN sin determinante. Las oraciones con tal estructura dan cuenta de las propiedades, calificación o juicios de valor relativos al núcleo nominal y, por ello, es posible que se combinen con mayor frecuencia con antecedentes indefinidos (qué tipo). He aquí ejemplos:

\footnotetext{
${ }^{3}$ Exponentes de definitud e indefinitud existenciales.
} 
Yo creo que se han resuelto asuntos que ya estaban muy maduros.

Nosotros los hombres podemos encontrar maneras de hacer cosas que parecían imposibles.

Muchas veces luchamos por libros que no son en absoluto como los que nos movieron a entrar en este negocio. $[=\text { no son así }]^{4}$

Parece que las oraciones en que se indica lugar favorecen la indefinitud del GN siempre que sus adjuntos revelen una localización espacial imprecisa:

Urge, por tanto, eliminar almacenes que se encuentran en lugares inadecuados.

Pero: Urge, por tanto, eliminar los almacenes que se encuentran en la calle Alcalá.

De la afirmación anterior se puede desprender que el sustantivo antecedente tiende a permanecer indefinido con tal de que la subordinada de predicado verbal cuente con argumentos y adjuntos de carácter indefinido:

El término de análisis de decisión engloba a un amplio conjunto de procedimientos sistemáticos para analizar problemas que requieren decisiones complejas.

Juan Hormaechea ha hecho cosas inmensas que nadie puede ignorar.

Dentro de este grupo de enunciaciones virtuales es la negación incluida en la subordinada la que, por lo visto, favorece el operador nulo con especial contundencia:

La humanidad instruida se ve abordada a diario por millones de palabras [...] que aluden a libros que nunca se abrirán.

Que el señor Senillosa, por tanto, tenga la gentileza de abstenerse de involucrarnos en guerras que no hemos peleado.

Por el contrario, los argumentos de carácter definido (nombres propios, pronombres personales ${ }^{5}$, nombres comunes definidos), adverbios y frases adverbiales de lugar (aquí, allí, en la calle Alcalá), adverbios y frases adverbiales de tiempo (en 1960, el martes, hasta ayer $)^{6}$ tienden a generar la presencia del artículo definido LOS / LAS.

Esta construcción es mucho más frecuente en los textos que escribió Goytisolo hasta 1957.

${ }^{4}$ Los sustantivos pueden calificarse también mediante oraciones de estructura no copulativa siempre que aporten alguna característica de su antecedente nominal, p. ej. El joven infante se enfrentó de repente con asuntos que Felipe II resolvía con dificultad. [= asuntos difíciles]

${ }^{5}$ En especial tú y nosotros.

${ }^{6}$ Estos adverbios tienen un fuerte carácter demostrativo (MNGLE, 2010: 339; Leonetti, 2012: 295). 
Ha de señalarse que reconocemos por definidos los argumentos y adjuntos de la subordinada actualizados mediante artículos definidos, demostrativos, posesivos prenominales y cuantificadores fuertes (para más información vid. Lyons, 1999: 17; Gutiérrez Rodríguez, 2008: 300, 308).

\section{Examen estadístico preliminar}

Procedemos a comprobar las hipótesis que hemos planteado con anterioridad. Añadimos otras dos variables estadísticas, tiempos gramaticales y función del antecedente (sujeto / objeto), observables en el material elaborado. A este efecto, nos servimos del corpus CREA en el dominio que recoge solo los textos de España. Sometemos a examen cuatro frases de entrada con sustantivos plurales (PALABRAS QUE, OPINIONES QUE, ASUNTOS QUE y LIBROS QUE) que anticipan potencialmente una subordinada de relativo siguiente, p. ej. ... comuniqué a los cuadros las palabras que me había dirigido el general / ... encontrar libros que hablan de conciencia cuántica o de psicología cuántica ${ }^{7}$. Los datos que esperamos obtener son variados y afectan a factores que, como sospechamos, responden de la referencia de los GN revisados. Hemos recogido 50 ejemplos de frases que contienen las estructuras mencionadas arriba, lo que representa un total de 200 ejemplos. Lamentablemente, como podíamos esperar, no todos los grupos van precedidos del AD o cero, operadores en que enfocamos nuestra atención. A parecen ocasionalmente otros determinantes (posesivos, demostrativos, indefinidos, numerales), que no hemos tomado en cuenta (45 ejemplos). Se ha comprobado que la presencia del artículo unos (calificado por algunos como cuantificador indefinido) es muy escasa en el corpus recopilado (7 ejemplos). Sin embargo, este cuantificador no es objeto de estudio en la presente elaboración y lo hemos desatendido. En suma, han quedado 151 ejemplos para analizar y comentar ${ }^{8}$. Una prueba numéricamente modesta, pero representativa, dada la amplia variedad estilística de textos que se han documentado. Para confirmar los resultados obtenidos se necesita naturalmente un tratamiento más profundo del tema. Abajo vemos un cuadro que presenta los diferentes datos que se han podido extraer del corpus seleccionado. Todos ellos dan

\footnotetext{
${ }^{7}$ La primera con antecedente objeto, y la segunda con antecedente sujeto.

${ }^{8}$ Otros cuatro ejemplos no representan la estructura oracional esperada.
} 
cuenta de la frecuencia absoluta de aparición de distintas categorías que creemos responsables de la (in)definitud de la expresión estudiada:

\begin{tabular}{|c|c|c|c|c|c|c|c|c|c|c|}
\hline \multirow{2}{*}{ Variables } & \multicolumn{2}{|c|}{ Palabras que } & \multicolumn{2}{|c|}{$\begin{array}{c}\text { Opiniones } \\
\text { que }\end{array}$} & \multicolumn{2}{|c|}{ Asuntos que } & \multicolumn{2}{|c|}{ Libros que } & \multicolumn{2}{|c|}{ Total 151} \\
\hline & las & cero & las & cero & los & cero & los & cero & $\begin{array}{l}\text { los / } \\
\text { las }\end{array}$ & cero \\
\hline $\begin{array}{l}\text { Número de } \\
\text { ocurrencias }\end{array}$ & 18 & 15 & 28 & 12 & 29 & 11 & 28 & 10 & 103 & 48 \\
\hline Negación & 0 & 4 & 0 & 0 & 3 & 3 & 0 & 1 & 3 & 8 \\
\hline $\begin{array}{l}\text { Estructura } \\
\text { copulativa }\end{array}$ & 0 & 3 & 0 & 2 & 3 & 4 & 0 & 1 & 3 & 10 \\
\hline $\begin{array}{l}\text { Elementos } \\
\text { definidos }\end{array}$ & 11 & 3 & 19 & 5 & 19 & 4 & 20 & 4 & 69 & 16 \\
\hline $\begin{array}{l}\text { Tiempos: } \\
\text { Presente }\end{array}$ & 14 & 12 & 24 & 11 & 15 & 9 & 18 & 9 & 71 & 41 \\
\hline Imperfecto & 2 & 2 & 3 & 1 & 11 & 2 & 2 & 0 & 18 & 5 \\
\hline PPS & 1 & 0 & 1 & 0 & 1 & 0 & 4 & 1 & 8 & 1 \\
\hline PPC & 1 & 0 & 0 & 0 & 1 & 0 & 2 & 0 & 4 & 0 \\
\hline $\mathrm{PP}$ & 0 & 0 & 0 & 0 & 1 & 0 & 2 & 0 & 3 & 0 \\
\hline Futuro & 0 & 1 & 0 & 0 & 0 & 0 & 0 & 0 & 0 & 1 \\
\hline Total & 18 & 15 & 28 & 12 & 29 & 11 & 28 & 10 & 103 & 48 \\
\hline $\begin{array}{l}\text { Antecedente } \\
\text { sujeto }\end{array}$ & 10 & 14 & 10 & 9 & 24 & 9 & 12 & 9 & 56 & 41 \\
\hline
\end{tabular}

A continuación sigue un cuadro análogo con los porcentajes que cada tipo de variable representa en términos de frecuencia relativa. Repárese en que el número de ocurrencias de los GN encabezados por los / las (103 casos) es mucho mayor que el de los que van sin determinante (48 casos). Es, por ello, necesario el cálculo de porcentajes para poder comparar objetivamente la frecuencia de eventos en las dos magnitudes desiguales (Müller, 1973: 132).

\begin{tabular}{|l|c|c|c|c|c|c|c|c|c|c|}
\hline \multirow{2}{*}{$\begin{array}{c}\text { Variables } \\
\text { (en \%) }\end{array}$} & \multicolumn{2}{|c|}{ Palabras que } & \multicolumn{2}{|c|}{ Opiniones que } & \multicolumn{2}{|c|}{ Asuntos que } & \multicolumn{2}{|c|}{ Libros que } & \multicolumn{2}{|c|}{ Promedio } \\
\cline { 2 - 13 } & las & cero & las & cero & los & cero & los & cero & $\begin{array}{c}\text { los / } \\
\text { las }\end{array}$ & cero \\
\hline $\begin{array}{l}\text { Número de } \\
\text { ocurrencias }\end{array}$ & 54,5 & 45,5 & 70 & 30 & 72,5 & 27,5 & 73,7 & 26,3 & $(67,7)$ & $(32,3)$ \\
\hline Negación & 0 & 26,6 & 0 & 0 & 10,3 & 27,3 & 0 & 10 & 2,6 & 16 \\
\hline $\begin{array}{l}\text { Estructura } \\
\text { copulativa }\end{array}$ & 0 & 20 & 0 & 16,6 & 10,3 & 36,4 & 0 & 10 & 2,6 & 20,7 \\
\hline
\end{tabular}


Janusz Pawlik

\begin{tabular}{|l|r|r|r|r|r|r|r|r|r|r|}
\hline $\begin{array}{l}\text { Elementos } \\
\text { definidos }\end{array}$ & 61,1 & 20 & 67,8 & 41,6 & 65,5 & 36,4 & 71,4 & 40 & 66,4 & 34,5 \\
\hline $\begin{array}{l}\text { Tiempos: } \\
\text { Presente }\end{array}$ & 77,7 & 80 & 85,7 & 91,7 & 51,7 & 81,8 & 64,3 & 90 & 70 & 86 \\
Imperfecto & 11,3 & 13,3 & 10,7 & 8,3 & 37,8 & 18,2 & 7,1 & 0 & 16,7 & 9,9 \\
PPS & 5,5 & 0 & 3,6 & 0 & 3,5 & 0 & 14,4 & 10 & 6,7 & 2,5 \\
PPC & 5,5 & 0 & 0 & 0 & 3,5 & 0 & 7,1 & 0 & 4 & 0 \\
PP & 0 & 0 & 0 & 0 & 3,5 & 0 & 7,1 & 0 & 2,6 & 0 \\
Futuro & 0 & 6,7 & 0 & 0 & 0 & 0 & 0 & 0 & 0 & 1,7 \\
Total & 100 & 100 & 100 & 100 & 100 & 100 & 100 & 100 & 100 & 100 \\
\hline $\begin{array}{l}\text { Antecedente } \\
\text { sujeto }\end{array}$ & 55,5 & 93,3 & 35,7 & 75 & 82,7 & 81,8 & 42,8 & 90 & 54,2 & 85 \\
\hline
\end{tabular}

¿Cuál es la información que se puede sacar de las cifras expuestas? Limitémonos a lo esencial que se encuentra en la columna de la derecha. La negación en las subordinadas precedidas del operador nulo aparece por término medio en un $16 \%$ de los casos, comparado con un 2,6\% referido a las subordinadas con artículo definido. Una diferencia apreciable que viene a confirmar nuestras previsiones. Lo mismo sucede en el caso de estructuras copulativas que se documentan en un $20,7 \%$ de las frases sin determinante, respecto a tan solo un 2,6\% testimoniadas en las de carácter definido. Por el contrario, los GN con determinante superan acusadamente a los grupos escuetos en cuanto al número de elementos definidos que abarcan $(66,4 \%$ vs $34,5 \%)$. La diferencia en otras variables (tiempos verbales, función sintáctica del antecedente) no parece tan decisiva. Aun así, el número de ocurrencias del pretérito perfecto simple es casi tres veces mayor $(6,7 \%)$ en los grupos determinados que en los indeterminados (2,5\%). Se vislumbra asimismo una clara tendencia a usar más los tiempos perfectivos del pasado en las estructuras encabezadas por el artículo definido (13,3\% frente a 2,5\%). Son formas apropiadas para anclar a un referente no genérico en una situación pasada que reconocen los interlocutores.

En el corpus revisado se registran ejemplos que exigen el uso automático de los / las. Les pertenece, sobre todo, la construcción superlativa de relativo [que más V], p. ej., Allí colocó los libros que más usaba. Entre las piezas léxicas, constituyentes del GN, que inducen la lectura determinada del GN se halla también el adjetivo siguientes, que lo hace de forma regular. Lyons (1999: 9) hace referencia a este tipo de definitud generada con respecto al inglés. Enumera, aparte del ya comentado next, otros modificadores que generan construcciones de referencia definida: first, only, same (He is the [an*] only student who dislikes phonology). El castellano admite mayor flexibilidad al respecto, ya que los 
cuantificadores correspondientes (primeros, únicos, mismos) se muestran a veces compatibles con el indefinido unos. No obstante, su aparición en los textos ha probado ser escasísima.

\section{Observaciones finales}

Nuestro estudio tiene un carácter preliminar y cuenta con varias restricciones. No tomamos en consideración, por ejemplo, las oraciones cuyos antecedentes desempeñan otras funciones que las de sujeto y objeto directo. No prestamos atención alguna a construcciones introducidas por otros relativos distintos a que (quienes, los cuales, los que, etc.). Tampoco nos han interesado los sustantivos en singular, etc. Son limitaciones que nos hemos impuesto con plena conciencia, dados los límites de espacio a que debe atenerse la presente publicación.

Los datos estadísticos alcanzados a base del material recogido confirman en principio las tesis articuladas al principio y pueden dar impulso a investigaciones ulteriores. Se ha proporcionado además información básica concerniente a los tiempos verbales (subjuntivo e indicativo en conjunto) y la función sintáctica del antecedente en la oración adjetiva. La relación entre las variables propuestas y la (in) definitud del GN no está probada, como en cualquier análisis de tipo inductivo, pero las cifras arrojan unas tendencias bastante claras. Salvo casos aislados, la tendencia final se repite en cada uno de los cuatro segmentos examinados. Aun así, las conclusiones que se extraen del examen estadístico tienen un valor aproximativo. Se necesitan otros recuentos más avanzados. Adviértase, al mismo tiempo, que los rasgos semántico-pragmáticos de las construcciones revisadas son otra cuestión que requiere un análisis detenido (Szabolcsi, 1983: 97).

\section{Bibliografía}

GUTIÉRREZ RODRÍGUEZ, E. (2008). «Rasgos categoriales de los determinantes», Actas del XXXVII Simposio Internacional de la Sociedad Española de Lingüística, Pamplona, 297-309.

HAWKINS, J. A. (1978). Definiteness and Indefiniteness. A study in Reference and Grammaticality Prediction. London: Croom Helm. 
ITURRIOZ LEZA, J. L. (1996). «Los artículos y la operación de determinación», en I. BOSQUE (ed.), El sustantivo sin determinación. La ausencia de determinante en la lengua española. Madrid: Visor Libros, 339-385.

KLEIBER, G. (1981). «Relatives spécifiantes et relatives non spécifiantes», Le Français Moderne, 49, 216-233.

LEONETTI, M. (1999). Los determinantes. Madrid: Arco / Libros.

LEONETTI, M. (2012). «Indefiniteness and Specificity», en J. I. HUALDE, A. OLARREA y E. O'ROURKE (eds.), Handbook of Spanish Linguistics. Oxford: Blackwell, 285-305.

LEONETTI, M. (2016). «Determinantes y artículos», en J. GUTIÉRREZ-REXACH (ed.), Enciclopedia Lingüística Hispánica, vol. I. London: Routledge, 532-543.

LYONS, C. (1999). Definiteness. Cambridge: Cambridge University Press.

MARIMOTO, Y. (2011). El artículo en español. Madrid: Castalia.

MÜLLER, CH. (1973). Estadística lingüística. Madrid: Gredos.

MNGLE - Real Academia Española y Asociación de Academias de la Lengua Española (2010). Nueva gramática de la lengua española. Manual. Madrid: Espasa Libros.

NGLE - Real Academia Española y Asociación de Academias de la Lengua Española (2009). Nueva gramática de la lengua española. Madrid: Espasa Libros.

PAWLIK, J. (2001). Selección de problemas de gramática española. Poznań: Wydawnictwo Naukowe UAM.

QUIRK, R. et al. (1985). A Comprehensive Grammar of the English Language. New York: Longman.

SZABOLCSI, A. (1983). «The possessor that ran away from here», The Linguistic Review, III, 89-102.

WELLS, H. G. (1895). La Máquina del Tiempo [en línea] <http://Le Libros.org> También en: <http://planetalibro.net>, fecha de consulta: 15 de octubre de 2018. 